\title{
Detuning Mechanism and Frequency Tracking Algorithm for Wireless Power Transmission System
}

\author{
Baoying $\mathrm{Li}^{1, *}$, Guangxing Xiang ${ }^{1}$, Chen $\mathrm{Li}^{1,2}$ and Kejia Zang ${ }^{1}$ \\ ${ }^{1}$ School of Information Science and Engineering, Dalian Polytechnic University, Dalian 116034, China \\ ${ }^{2}$ School of Electrical Engineering and Telecommunications, University of New South Wales, UNSW Sydney, NSW 2052, Australia
}

Received 12 April 2017; Accepted 29 August 2017

\begin{abstract}
Wireless power transmission (WPT) uses a loosely coupled transformer to transmit electric energy. The resulting system's transmission efficiency and distance are affected by factors such as, inductance parameter and resonance frequency offset. To reduce the influence of detuning on transmission efficiency, a frequency tracking method based on the hill-climbing algorithm was proposed in this study. The WPT model was established to depict the detuning mechanism and the transmission efficiency affected by natural frequency changes in the primary and secondary coils. Experimental data were used to prove the correctness of the theoretical analysis. Results demonstrate that the coupling coefficient of the two coils is proportional to the increase of the distance between the transmitting and receiving coils. Hence, coupling coefficient influences transmission efficiency. Another important factor is distance $S$, as its increase leads to mutual loss, which breaks the previous state of the best resonance compensation, which in turn reduces the transmission efficiency of WPT. When distance $S$ increases from $10 \mathrm{~mm}$ to $30 \mathrm{~mm}$ and the system has no frequency tracking, the transmission efficiency decreases from $45 \%$ to $7.5 \%$. However, when distance $S$ increases from $10 \mathrm{~mm}$ to $30 \mathrm{~mm}$ and the system has frequency tracking, the transmission efficiency decreases from $70 \%$ to $19.1 \%$. When distance $S$ is equidistant, the transmission efficiency is much higher with than without frequency tracking. The proposed method can effectively raise the transmission efficiency of the WPT system and control the cost of hardware, which optimizes practical engineering design.
\end{abstract}

Keywords: Wireless power transmission, Detuning, Frequency tracking, Hill-climbing algorithm

\section{Introduction}

Wireless power transmission (WPT) is a new type of electric energy transmission technology. By using the electromagnetic induction principle, the power transmission between the power supply system and electrical equipment is completed without a physical connection [1-4]. Good security, high reliability, and flexibility are the advantages of WPT. Some special applications, such as coal mining, implantable bio-medical systems [5-6], and internal power supply in the wall have utilized WPT. Thus, WPT technology has widespread use, and the number of applications in wireless power transmitted equipment is gradually growing. Currently, WPT technology studies have substantially progressed, hence, companies have engaged in many aspects of exploring the system design, production, and applications of WPT.

In the past decade, domestic and international innovations on the WPT system have been fruitful. Wireless charging equipment increasingly changed people's daily lives, especially in terms of wireless mobile phone charging and wireless charging of an electric vehicle development.

\footnotetext{
*E-mail address: liby@dlpu.edu.cn

ISSN: 1791-2377 @ 2017 Eastern Macedonia and Thrace Institute of Technology. All rights reserved. doi:10.25103/jestr.104.14
}

With the development of research and applications, the requirements of transmission efficiency in WPT increased; however, detuning destabilizes frequency, which main causes transmission inefficiency [7-9]. Transmission efficiency pertains to the phenomenon wherein the system matches the power equipment requirements, thereby conserving energy. Therefore, improving frequency stability is an urgent problem.

Conventional resonate methods are mostly robust control ones based on hardware, which result in numerous disadvantages, such as complex design, expensive hardware, and inefficient transmission. A narrow transmission distance severely affects application in WPT. Accordingly, the use of microprocessors in software design instead of hardware design can effectively reduce costs and raise transmission efficiency.

Based on the above analysis, this study adopts the hillclimbing algorithm to ensure that the system functions in the vicinity of resonant frequency, thereby raising the transmission efficiency and output power of the system. Moreover, this research could effectively enhance the transmission efficiency and control hardware cost by exploring the effects of natural frequency changes in primary and secondary coils on transmission efficiency and analyzing the detuning mechanism. Therefore, hill-climbing algorithm has great practical value and extensive applications in the future. 


\section{State of the art}

Scholars have considerably examined WPT. Studies have focused on frequency stability, especially with regard to its control. Fu Wenzhen[10] proposed the use of the phaselocked loop method to achieve frequency automatic tracking, and the resulting tracking accuracy was relatively high. However, the tracking range was narrow, and frequency tracking could not be performed when the interference was too large. Qiang H.[11] and Sun Y.[12] suggested a technique that incorporated the tuning circuit in the launch circuit to achieve the primary side of the compensation capacitor with timely dynamic compensation when the output load changed. Consequently, the operating frequency was as consistent as the receiver resonant frequency, but such complex control method entailed costly hardware. Zhu C. B.[13] studied the loss of an electromagnetic resonance WPT system and indicated that under a loosely coupled condition, the system improved resonance quality, which could increase transmission efficiency of transmission; however, the influence of inductance parameters on transmission efficiency was not analyzed. Wang C. S.[14] examined equivalent circuit and power transmission capabilities of various topologies, but the model was inaccurate because of unknown parasitic parameters in the system. Qiang H.[15] discussed the optimal transmission efficiency of a WPT, but the studied topology was designed to be related to certain parameters, including load, operating frequency, and coil size. If various topologies were designed in the system, then the system would less predictable. Sample A. P.[16] proposed a new type of coupler topology that could produce a large coupling coefficient given an equal diameter to a conventional circular coupler; however, such typology involves increased complexity. Budhia M.[17] developed a simple equivalent circuit model of a deep-level resonant coupling system, explored three key factors (frequency, distance, and impedance matching) for determining transmission performance, and successfully charged a laptop to $0.7 \mathrm{~m}$ off a resonant coil. However, the charge resonant frequency was single, which might explain the possible detuning situation. Compared with the traditional inductive coupling scheme, the magnetic coupling resonator of WPT can more efficiently transmit power than the far field method, as proposed by Zhu C.[18], Zhen N. L.[19], Casanova J. J.[20], and Kim Y. H.[21]. However, fixed distance and direction limited their study, and the transmission efficiency rapidly decreased when the receiver was far from the optimum operating point. However, Villa J. L.[22] presented a topology improvement theory without a strict alignment necessary to achieve a highly efficient energy delivery. Kim J.[23] described the addition of loop switching techniques to multiple loop coils at the transmitter and load side to improve transmission efficiency. Each ring coil should be individually controlled, which increased design size and system complexity. Ricketts[24] used a digital tuned capacitor, which was limited in long distance variations. Chen C. J.[25] applied a capacitor compensation technique to achieve a wide range of power transmission. However, this method required a complex capacitive dynamic tuning to maintain resonance conditions. When the wireless power supply system is applied as a consumer product, the changes of the distance between the primary and secondary coils, the distance, and the load are extremely random. Therefore, ensuring the stability of the system operating frequency guarantees that the system maintains maximum efficiency output.

Based on the above analysis, a simple and effective frequency tracking method called hill-climbing algorithm is proposed. In addition, electromagnetic simulation software Maxwell is used to simulate the variation of coupling coefficients when distance, offset, and rotation are in different states. The experimental platform is established to simulate the coupling circuit. The influence of inductance parameters on the transmission efficiency is discussed. The changes of transmission frequency once frequency tracking are added into the system are also considered. By using experimental and theoretical means, this study aims to address the reduced transmission efficiency due to detuning, thereby benefiting engineering applications.

The remainder of this study is organized as follows. Section 3 presents the hill-climbing algorithm and the transmission efficiency in a WPT system. Section 4 discusses the influence of the transmission efficiency by changing the value of inductance parameters. This section also analyzes the output voltage waveform and the energy transmission efficiency with and without frequency tracking. The last section summarizes the conclusions.

\section{Methodology}

\subsection{Theory of inductive coupling system}

The operational principle of a non-contact WPT system is shown in Fig. 1.

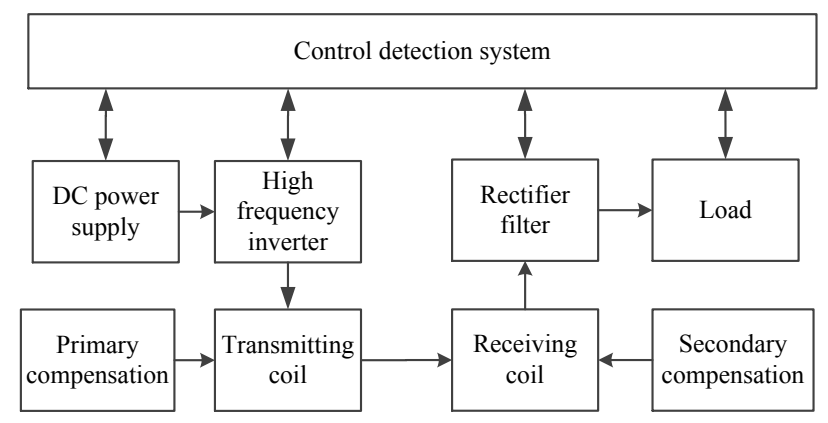

Fig. 1. Principle of a contactless power transmission system

A complete coupling WPT system mainly includes a power supply, inverter circuit, primary side compensation and transmitting coil, secondary side compensation and receiving coil, high frequency contravariant, the filter, and voltage regulator.

Compared with traditional transformers, an inductive coupling system transports power based on the model of a loosely coupled transformer. Furthermore, the air magnetic circuit is larger between the transmitter and receiver. An air gap generally increases the resistance of a magnetic circuit. From Ohm theorem of the magnetic, most magnetomotive force is distributed on the air gap magnetic circuit, which mainly causes transport inefficiency. A substantial amount of reactive power is consumed by the original coil, which can help alter receiver power, thereby reducing system requirements for power by accessing the compensation capacitor. 


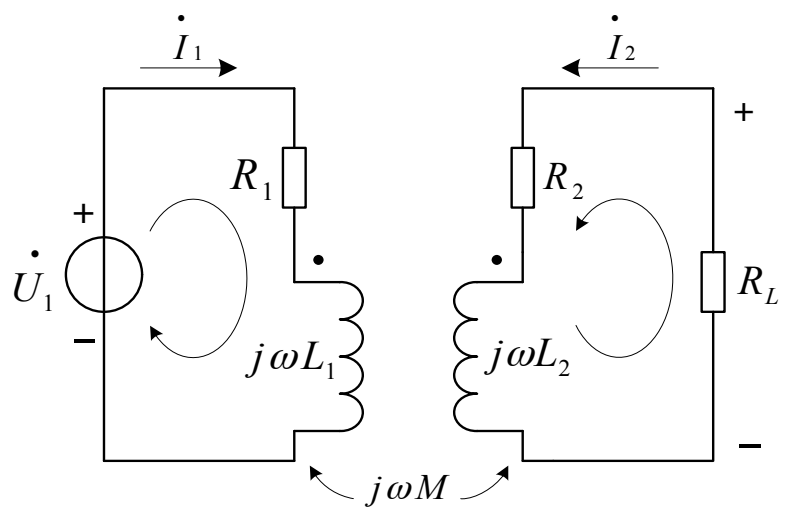

Fig.2 (a). Basic circuit model of a loosely coupled transformer

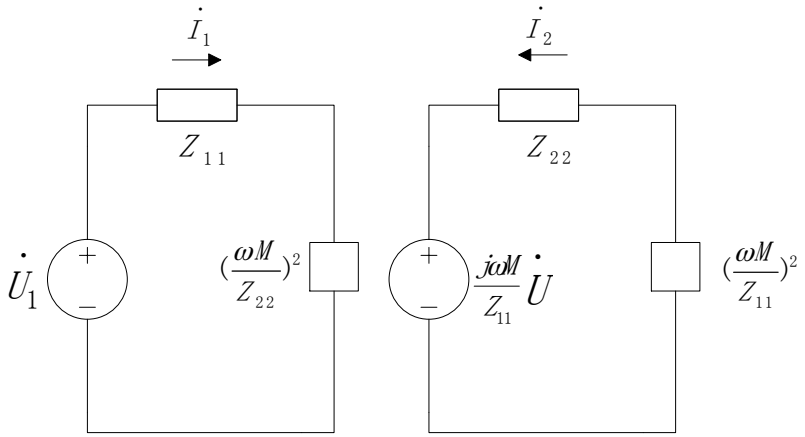

Fig.2 (b). Former vice edge decoupling of a loosely coupled transformer

The Fig. 2(a) shows a basic transformer circuit. For the primary side, the power is $\dot{U}_{1}$, the current is $\dot{I}_{1}$, the selfinductance is $L_{1}$, and inductance internal resistance is $R_{1}$ (conductor resistance is ignored). For the secondary side, the current is $\dot{I}_{2}$, the self-inductance is $L_{2}$, the inductance internal resistance is $R_{2}$, the load resistance is $R_{L}$, and the former mutual inductance is $M$. According to the Kirchhoff Voltage Law (KVL) circuit, the loop equation is established as follows:

$\left(j \omega L_{1}+R_{1}\right) \dot{I}_{1}+j \omega M \dot{I}_{2}=\dot{U}_{1}$

$j \omega M \dot{I}_{1}+\left(R_{L}+R_{2}+j \omega L_{2}\right) \dot{I}_{2}=0$

The above equations are composed of two independent circuit equations (primary and secondary sides) connected by mutual inductance, which is the basic principle to analyze the performance of a voltage transformer. The equation $Z_{11}=R_{1}+j \omega L_{1}$ is the primary loop impedance; the equation $Z_{22}=R_{2}+j \omega L_{2}+R_{L}$ is the secondary loop impedance; and the equation $Z_{\mathrm{M}}=j \omega \mathrm{M}$ is the interaction inductive reactance. The aforementioned equations can be abbreviated as follows:

$$
\begin{aligned}
& Z_{11} \dot{I}_{1}+Z_{M} \dot{I}_{2}=\dot{U}_{1} \\
& Z_{M} \dot{I}_{1}+Z_{22} \dot{I}_{2}=0
\end{aligned}
$$

The Former side decoupling:

$$
\begin{aligned}
& \dot{U}_{1}=\left(Z_{11}-\frac{Z_{M}{ }^{2}}{Z_{22}}\right) \dot{I}_{1} \\
& \frac{Z_{M}}{Z_{11}} \dot{U}_{1}=-\left(Z_{22}-\frac{Z_{M}{ }^{2}}{Z_{11}}\right) \dot{I}_{2}
\end{aligned}
$$

\subsection{Analysis of transmission efficiency}

The equivalent circuit of the transformer is shown in Fig. 2(b). This figure indicates Equations (5) and (6), and Equations (7) and (8) as follows:

$$
\begin{aligned}
& \dot{I}_{1}=\frac{\dot{U}_{1}}{Z_{11}-Z_{M}{ }^{2} Y_{22}}=\frac{\dot{U}_{1}}{Z_{11}+(\omega M)^{2} Y_{22}} \\
& \dot{I}_{2}=-\frac{Z_{M}}{Z_{22}} \dot{I}_{1}=-\frac{j \omega M \dot{U}_{1}}{Z_{11} Z_{22}+(\omega M)^{2}}=-\frac{j \omega M Y_{11} \dot{U}_{1}}{Z_{22}+(\omega M)^{2} Y_{11}}
\end{aligned}
$$

In Equation (7), the reflected impedance is $(\omega \mathrm{M})^{2} \mathrm{Y}_{22}$. The equivalent impedance reflects the impedance in the secondary circuit by the mutual inductance to the primary loop. The character of the reflected impedance is opposite to $Z_{22}$, and the inductive-capacitive is transformed into capacitive-inductive.

The SS capacitor compensation is operated in the primary and secondary sides to ensure that the system works with maximum transmission efficiency. The system should also work with zero-phase frequency in load impedance to reduce reactive power component and increase energy transmission efficiency. Hence, the primary and secondary loops work at the resonant frequency, and the system could also satisfy the equation below [26]:

$\omega L=\frac{1}{\omega C}$

The circuit is a pure resistive circuit, and the equivalent circuit is shown in Fig. 3:
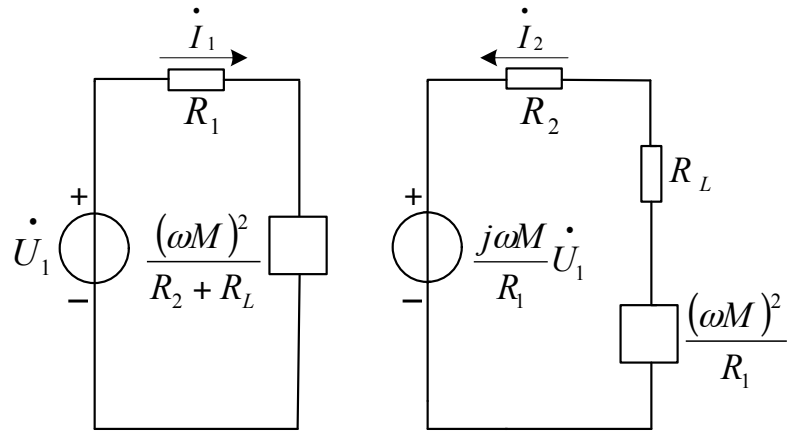

Fig. 3. Primary and secondary sides decoupling model of the resonance state of the transformer

The input power on the primary side coil $\mathrm{L}_{1}$ is:

$\mathrm{P}_{1}=\dot{\mathrm{U}}_{1} * \dot{I}_{1}=\frac{\left(R_{2}+R\right) U_{1}^{2}}{R_{1}\left(R_{2}+R\right)+(\omega M)^{2}}$

The output power on the secondary side load $\mathrm{R}_{1}$ is: 
$\mathrm{P}_{2}=\dot{\mathrm{U}}_{2} * \dot{I}_{2}=\frac{R(\omega M)^{2} U_{1}^{2}}{\left[R_{1}\left(R_{2}+R\right)+(\omega M)^{2}\right]^{2}}$

In Equation (11):

$$
\dot{U}_{2}=\frac{R(\omega M)^{2} \dot{U}_{1}}{R_{1}\left(R_{2}+R\right)+(\omega M)^{2}}
$$

Transmission efficiency is:

$\eta=\frac{P_{2}}{P_{1}}=\frac{(\omega M)^{2} R}{\left[R_{1}\left(R_{2}+R\right)+(\omega M)^{2}\right]\left(R_{2}+R\right)} \times 100 \%$

According to Equation (13), transmission efficiency is related to the resistances of the primary and secondary inductance, as well as the mutual inductance, the load resistance, and the operating frequency in the system. The inductance internal resistance is also connected to the wire, wire diameter, and working frequency. Under this circumstance, an equal number of coils in the primary side ( $R_{1}=R_{2}=R_{0}$ ) can be obtained. In the high-frequency situation, the internal resistance of the coil increases because of the skin effect of the conductor. Thus, multiple copper wires can be selected, and a coil made of a silver-plated copper conductor or a Liz line can be made to reduce the internal resistance of coil $R_{O}$ to enhance power transmission efficiency. If $R>R_{O}$, then the transmission efficiency is close to $100 \%$. Mutual inductance $M$ is associated with the coil radius, turns, and magnetic core material, as well as the air gap thickness between the primary and secondary loops [27-29].

\subsection{Realization of the hill-climbing algorithm}

During WPT, the system is subjected to three factors: the external interference, the temperature drift of the source capacitor, and the temperature drift of the secondary terminal capacitor. Hence, the resonance frequency of the transmitting and receiving system is changed. The main cause of the shift of this design's resonance point is the gap between the primary and secondary coils. Therefore, with the realization of the maximum transmission efficiency as the starting point, this study analyzes the interference from the receiver frequency of detuning factors and suggests frequency tracking for maximum efficiency technology to raise transmission efficiency and system stability and to address robust resonance frequency. Thus, when the distance between the primary and secondary coils are changed, the resonant frequency would also be changed, and the system should be based on the output side of the receiver to adjust the output pulse width modulation (PWM) driving frequency in order to adapt to external changes and maintain maximum power transmission efficiency[30] .

The principle of the hill-climbing algorithm indicates that a small perturbation of input disturbance mixed in the system can reduce output power. When a fixed amount of variation is allowed to control the system's output frequency, estimating the power change in the load receiving terminal is necessary to ensure the increase or decrease of the output frequency in the control system. This frequency is then converted as a reference for the control system, and the process is repeated to achieve maximum load acceptance power.
The hill-climbing principle is depicted in Fig.4. When the output load power changes, the transmission efficiency is also altered. If the transmitter operating frequency moves from operating point $\mathrm{A}$ to $\mathrm{B}$, then $\Delta f>0$ and $\Delta \eta>0$ and the frequency continues to increase along the original direction. If the transmitter operating frequency moves from operating point $\mathrm{B}$ to $\mathrm{A}$, then $\Delta f<0$ and $\Delta \eta<0$ and the frequency continues to increase along the opposite direction. If the transmitter operating frequency moves from operating point $\mathrm{E}$ to $\mathrm{D}$, then $\Delta f<0$ and $\Delta \eta>0$, then the frequency continues to decrease along the original direction. If the transmitter operating frequency moves from operating point $\mathrm{D}$ to $\mathrm{E}$, then $\Delta f>0$ and $\Delta \eta<0$ and the frequency decreases along the opposite direction. By repeating the above process, the best resonance point can be identified and the optimized output can be realized. The accuracy of the hill-climbing algorithm is decided by $\Delta f$; thus, the program can be conveniently directly modified.

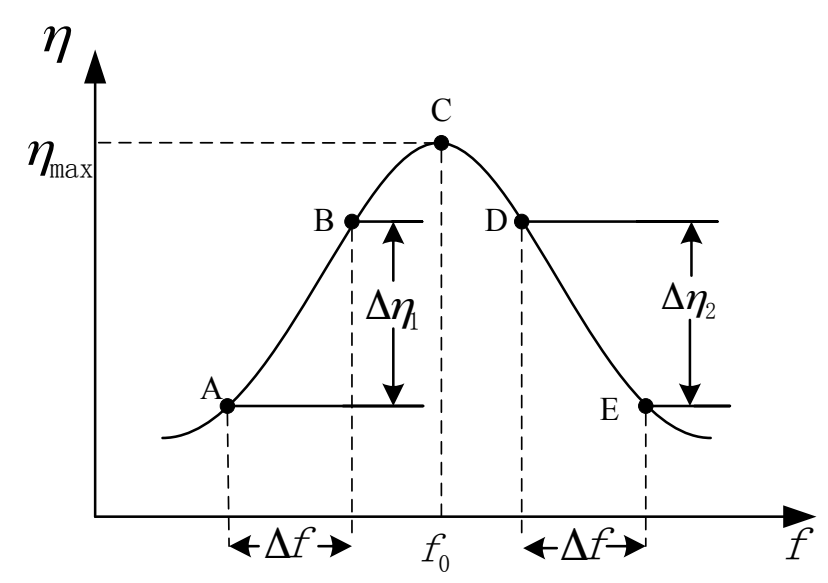

Fig. 4. Schematic of the climbing algorithm

\subsubsection{Hardware Design}

This system uses STM32 as the control core, establishing a wireless power supply system with the loosely coupled transformer. STM32 generates two complementary PWM signals (dead time 2us), and this signal could drive the $\mathrm{H}$ bridge inverter after IR2110 uplift voltage. After receiving a high frequency rectification filter, the LM2596 realizes the adjustable voltage regulation and produces outputs to the load.

As shown in Fig. 5, STM32 generates two complementary PWM waveforms. Once the IR2110 is loaded to STM32, the system generates four output PWM waveforms, which drives the back stage $\mathrm{H}$ bridge circuit. $\mathrm{R}_{1}$ indicates the small power sampling resistor using Constantan wire [31].

The real-time detection of the transmission loop's current is required with the real-time detection of its resonant state. The commonly used current detection methods include the current transformer and small resistance sampling methods. The smaller the resonance offset, the less accurate the current transformer sampling of the true response of the resonance offset. However, the system cannot make an actual judgment in this state, and thus, the Constantan wire is selected to sample the small sinusoidal current waveform, and the back stage is amplified.

As the Constantan wire is disconnected from the ground, the collection of the small sinusoidal signal is a differential signal and must be amplified by differential amplification 
and then connected to the back stage circuit. Thus, $R_{2}=R_{3}$ amplification. and $R_{4}=R_{5}$ are known by the principle of differential

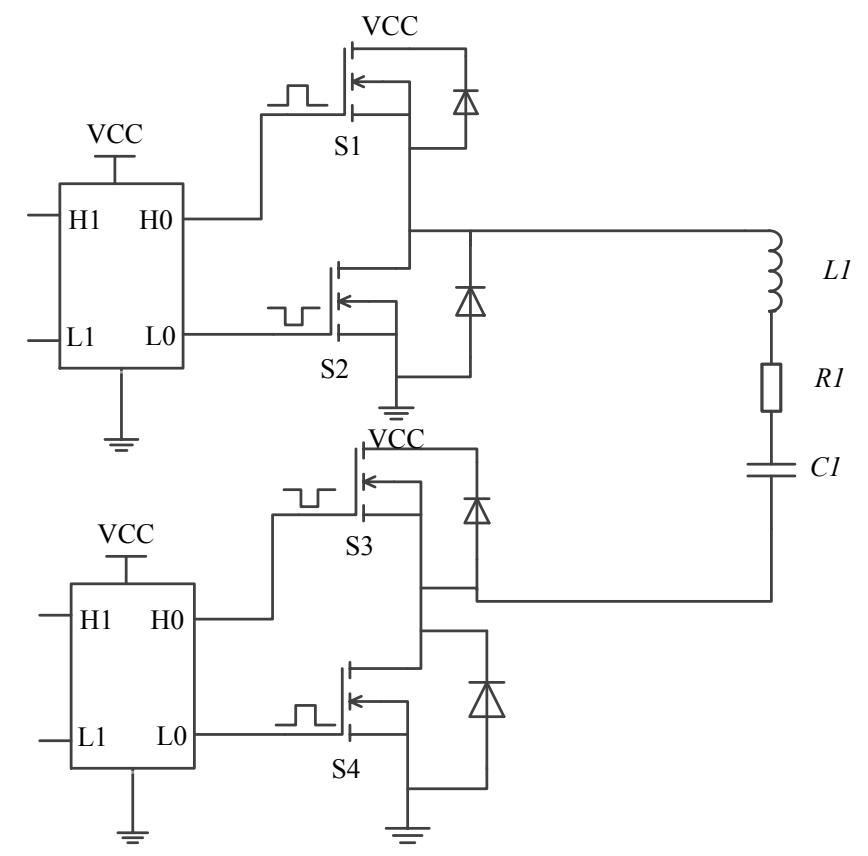

Fig. 5. Schematic of the inverter circuit of $\mathrm{H}$-bridge

In Fig. 6(a), after differential amplification of the signal, the signal is a high-frequency AC small signal without isolation, and $\mathrm{AD}$ sampling cannot be processed directly. Direct sampling is inaccurate and cannot truly reflect the current in the resonator. Fig. 6(b) demonstrates that Vol is the resonant small-current differential amplification voltage, which is rectified to obtain a DC voltage as the input voltage Vs of following the voltage. Thus, Vs varies in direct proportion to the detected current amplitude. Voltage follower has extremely high input impedance with almost no signal source drain current. The value of output impedance is extremely low, and the voltage follower has extremely low output impedance. To decrease internal voltage when the output current is backward, the voltage follower can be regarded as a voltage source to ensure authentic $\mathrm{AD}$ sampling[32]. The physical map of the system is shown in Fig. 7.

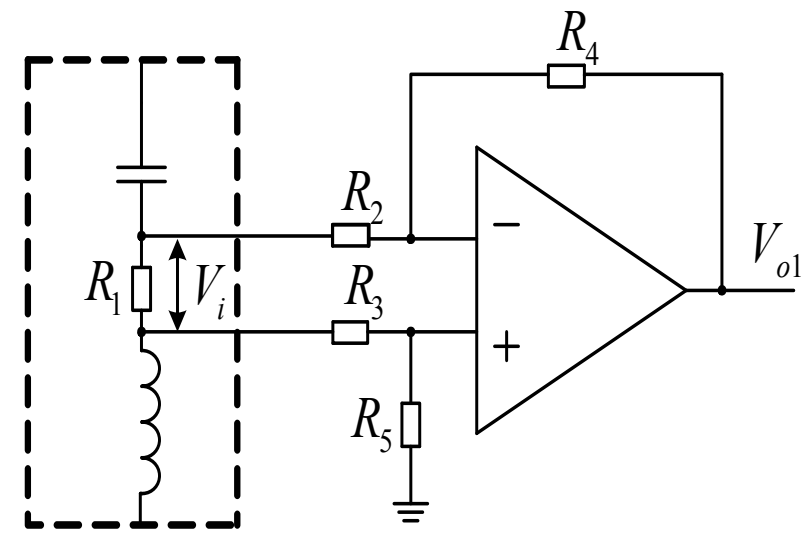

Fig.6 (a). Principle of high current sampling

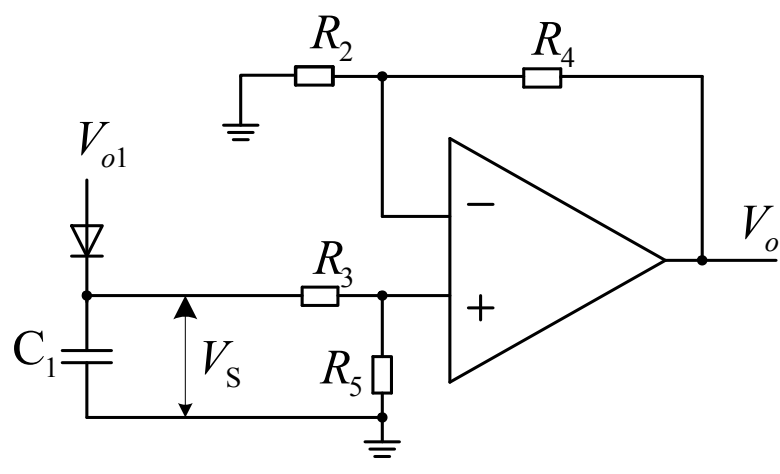

Fig.6 (b). Diagram of the principle of high frequency small signal rectifier and isolation

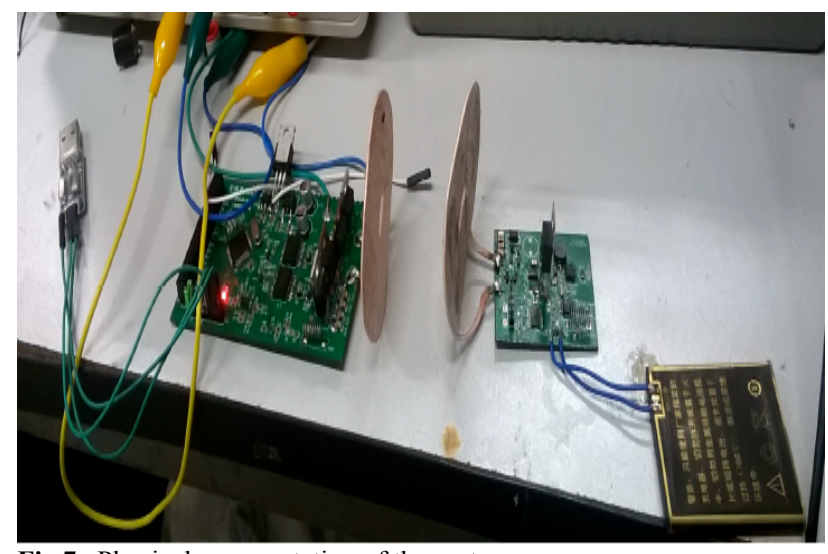

Fig.7. Physical representation of the system

\subsubsection{Software Design}

As the system is based on hill-climbing algorithm with frequency tracking, it is easy to control and implement and allows for cost-effective hardware. However, the slight disturbance alters the system transmission efficiency $\eta$ to shift near the optimum resonance point, leading to an unstable state and a possible increase in the system failure rate. Therefore, this study proposes a method to stabilize the 
system based on the hill-climbing algorithm. According to such method, when the external interference changes the system transmission efficiency $\Delta \eta$, that is, if $\Delta \eta>a$, then the hill-climbing algorithm is performed, the disturbance parameters $\Delta f$ are added into the system, and the resonant frequency is changed. If $\Delta \eta<a$, then the system retains the previously unchanged resonant frequency $f$, and then scans it every $\Delta t_{1}$ time. Thus, the system makes judgments to adjust when greater interference occurs, and the above algorithm lacks rigor. Moreover, the scan results are always $\Delta \eta<a$ when the external interference is slowly added into the system, and the direction of change is always the same. Consequently, the transmission efficiency of the system gradually diminishes, and thus, program designs must add additional correction links. The system unconditionally joins the maximum power point $\Delta f$ to reset operation every $\Delta t_{2}$ in order to adjust to the maximum power point, and the above process continuously repeats until the power is completely adjusted[33-34].

\section{Result Analysis and Discussion}

\subsection{Influence of inductance parameters on transmission efficiency}

In this experiment, PC74 ferrite core (Permeability is 2000) is used as a loosely coupled transformer core. The study discusses the factors that influence the coupling coefficient of the loose coupling transformer, which uses Ansoft Maxwell 3D software and tests inductance and mutual inductance values from the air gap thickness, lateral, vertical lateral, and rotational four aspects of the simulation.

The simulation results of inductance and mutual inductance are close to the actual measured values. The system also uses air gap thickness $\mathrm{d}=10 \mathrm{~mm}$ as the measurement datum. The errors are 3.01\% (inductance) and $2.42 \%$ (mutual inductance), and the reliability of simulation is verified by using Ansoft Maxwell 3D software.

Fig. 8(a) depicts the results of using the Ansoft Maxwell $3 \mathrm{D}$ to design a loosely coupled transformer with the same size as the actual model and a Y-axis as the center. The original secondary coil turns (23 turns) are set to simulate the WPT system (the number of turns with the actual model). Each turn wire current is $1 \mathrm{amp}$. Finally, this study calculates primary and mutual inductance. The magnetic flux density B's vector field diagram is shown in Fig. 8(b).

Inductance and mutual inductance are calculated under different conditions of air gap thickness $(2,4,6,8$, and 10 $\mathrm{mm})$, axial deviation $(2,4,6,8$, and $10 \mathrm{~mm})$ and deflection angles $(10,20,30$, and 40 degree).

As shown in Fig. 9, inductance and mutual inductance decrease rapidly as air gap thickens, and the coupling degree slightly decreases, demonstrating that inductance and mutual inductance are sensitive to the change of air gap thickness. The values of inductance and mutual inductance gradually decrease, and the coupling degree is not evident in this study, which indicates that the inductance and mutual inductance are not sensitive to lateral and rotational changes. Therefore, inductance and mutual inductance are influenced slightly by lateral deviation and rotation.

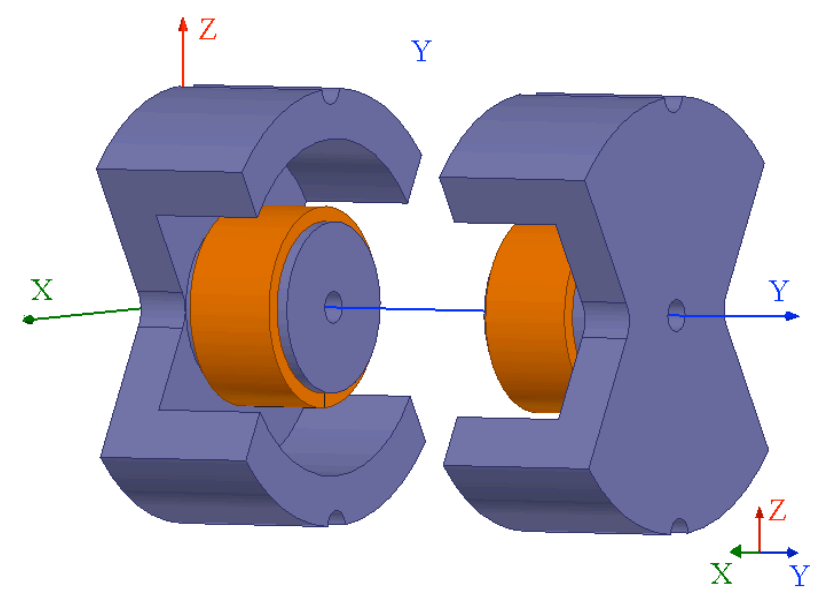

Fig.8 (a). Basic model of loosely coupled transformer

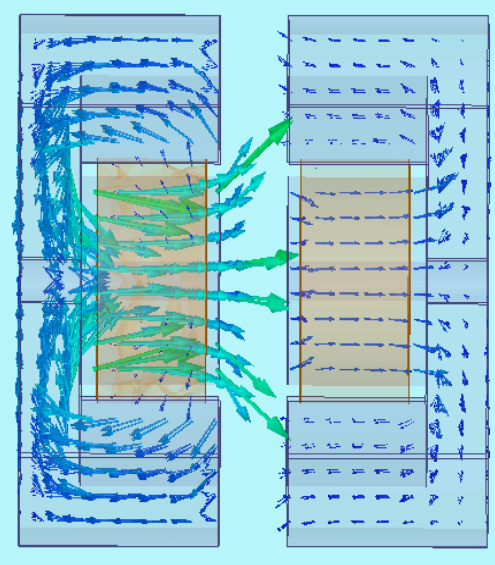

Fig.8 (b). Vector graphic of the magnetic flux density

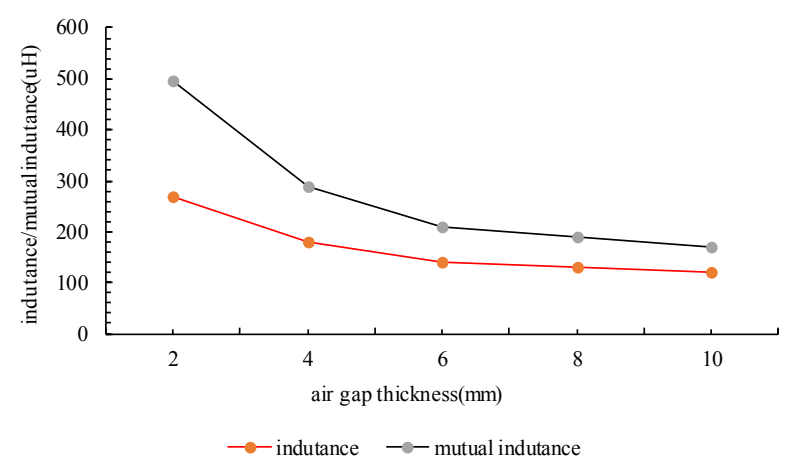

Fig.9 (a). Influence of air gap thickness on mutual inductance

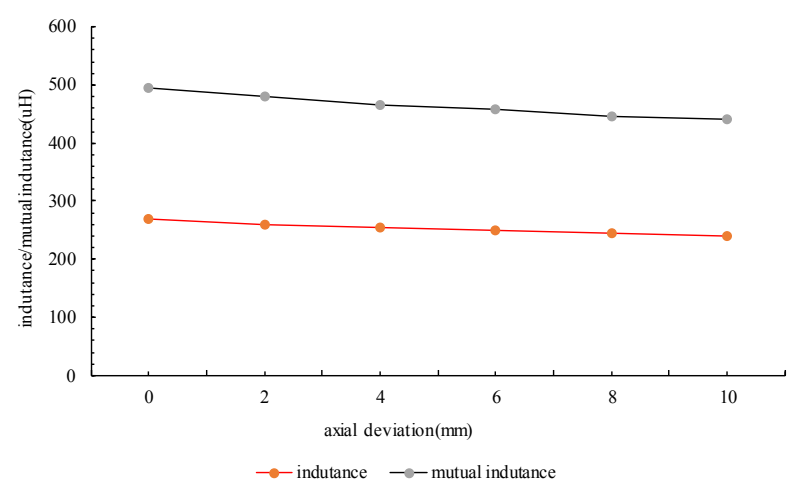

Fig.9 (b). Influence of axial deviation on mutual inductance 


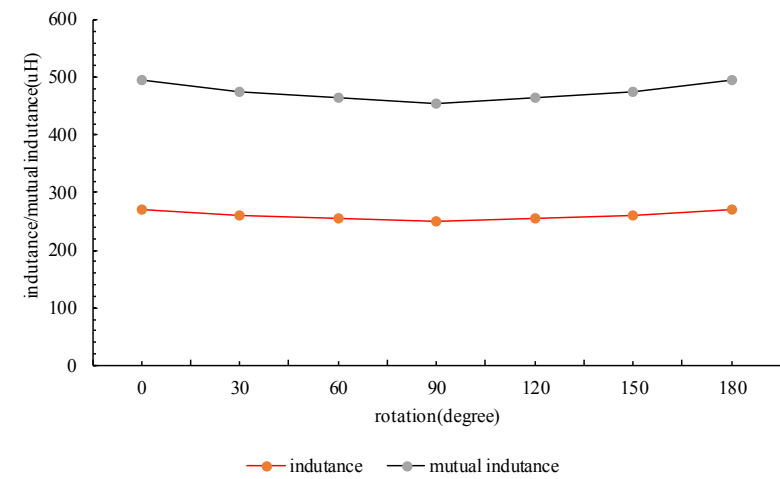

Fig.9(c). Influence of rotation on mutual inductance

Fig.9. Influence of air gap, axial deviation, and rotation on mutual inductance

4.2 Analysis of the experimental results for output voltage and transmission efficiency in the performance of wireless power transmission

A $20 \mathrm{~V}$ constant current output voltage source is selected as the DC power supply. When the values of the circuit parameters are similar, no frequency tracking and frequency tracking load output voltage curves are shown in Fig. 10(a). The receiver is not rectified and regulated, so the output is a sine wave and the peak value is up to $5 \mathrm{~V}$. When the frequency tracking structure is added into the system, the load output voltage curve is shown in Fig. 10(b), and the peak-to-peak value is $7.5 \mathrm{~V}$. Compared with each other, the WPT system with a frequency tracking structure output is more powerful. High transmission efficiency and the waveform are not prone to distortion, and the system is more stable and reliable.

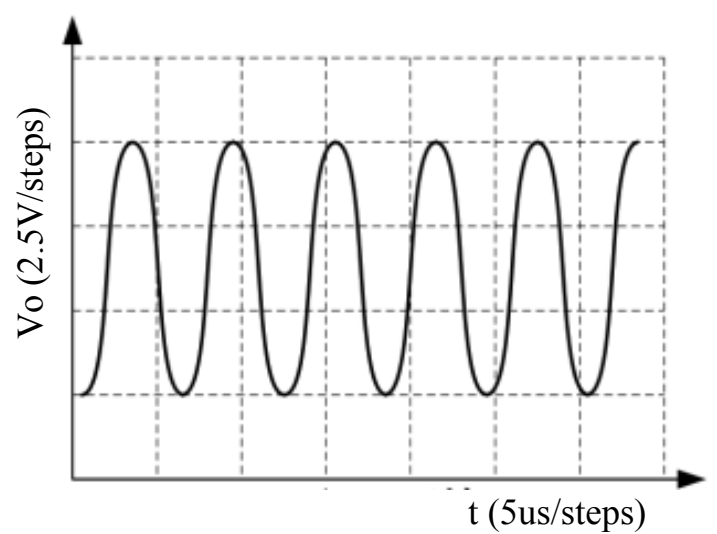

Fig.10 (a). Output voltage waveform of load without frequency tracking

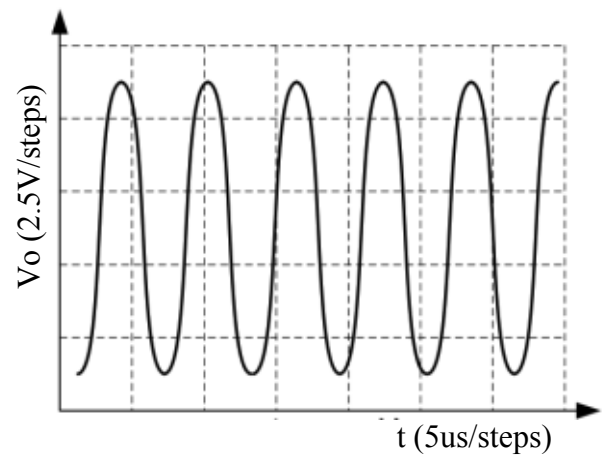

Fig.10 (b). Output voltage waveform of load with frequency tracking
Fig. 11 shows the power transfer efficiency with and the without tracking system. Experimental results demonstrate that when the distance $S$ between two coupling coils increases, the coupling coefficient $\mathrm{k}_{12}$ is a function of distance $S$. The first experimental variable is distance $S$, as shown in the figure, as it changes the transmission efficiency of the system. When distance $S$ changes from $5 \mathrm{~mm}$ to 40 $\mathrm{mm}$, the overall system transmission efficiency could be calculated. When distance $S$ is over $40 \mathrm{~mm}$, the value of overall efficiency decreases.

In sum, efficiency decreases slowly when frequency tracking is added in the system. At this point, the main factor affecting the transmission efficiency is coupling coefficient. Another important factor is that when distance $S$ increases, the mutual inductance decreases, which breaks the previous state of the best resonant compensation and reduces transmission efficiency. When $S=10 \mathrm{~mm}$, system transmission efficiency increased from $45.3 \%$ to $70.0 \%$ $(1.39 \mathrm{~dB})$. When $S=20 \mathrm{~mm}$, transmission efficiency increased from $21.8 \%$ to $38.4 \%(1.22 \mathrm{~dB})$. Finally, when $S=$ $30 \mathrm{~mm}$, transmission efficiency increased from $7.5 \%$ to $19.1 \%(1.06 \mathrm{~dB})$. The experimental results are consistent with the simulation results, proving that the frequency tracking technology in this study is reliable[35-36].

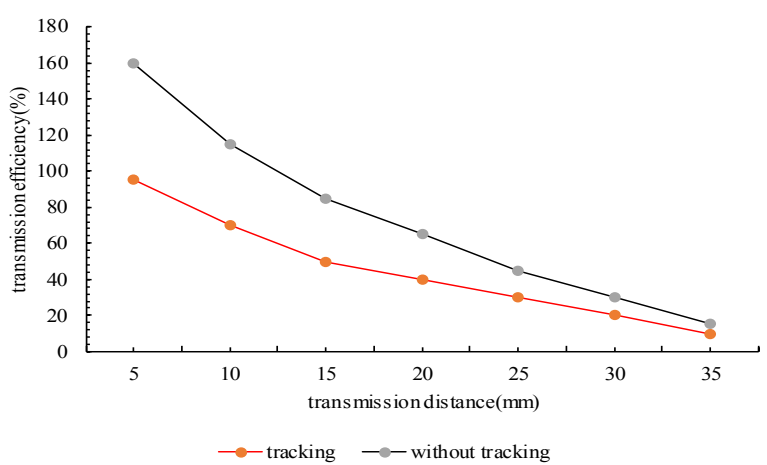

Fig.11. Transfer efficiency curve with and without tracking

\section{Conclusions}

To explore the influence of detuning on the power transmission efficiency in a WPT system, this study examined the factors that affected the transmission efficiency of the system by establishing a WPT model. The physical model of a WPT system was then proposed, and a combination of numerical simulation and experimental research was adopted to analyze the frequency instability caused by efficiency and detuning in a WPT system. The following conclusions are drawn:

(1) In loosely coupled transformers, inductance and mutual inductance were affected by air gap thickness, but were less affected by lateral displacement and rotation.

(2) In comparing the absence and presence of frequency tracking in the load output voltage curve, when the system was in no frequency tracking node, the peak-to-peak value was $5 \mathrm{~V}$; when the system was in frequency tracking node, the peak-to-peak value was 7.5 V. The output power of WPT system was higher with a frequency tracking structure, the waveform was not prone to distortion, and the system was more stable and reliable.

(3) The coupling coefficient $\mathrm{k}_{12}$ was a function of distance $S$, such that when $S$ increased, overall system transmission efficiency decreased. When the frequency tracking based on 
the hill-climbing algorithm was added, system transmission efficiency decreased gradually as distance increased. Without tracking in the system, when distance $S$ increased from $10 \mathrm{~mm}$ to $30 \mathrm{~mm}$, then the transmission efficiency decreased by $83.4 \%$. When the system was tracked, the transmission efficiency was reduced by $72.7 \%$. When the distance was equal, the frequency tracking of the transmission efficiency increased. Therefore, it was effective for raising the transmission efficiency of the WPT system.

Based on both experiments and theoretical analysis, this study proposes a frequency tracking method based on hillclimbing algorithm, generation of a simple WPT system, reduction of hardware circuit costs, and production of conditions close to the actual situation, which has a certain demonstration effect on the follow-up development of the WPT system. However, the system has some limited experimental conditions, such as coil winding errors. Thus, future research could increase the system's transmission efficiency by further optimizing software and hardware.

This is an Open Access article distributed under the terms of the Creative Commons Attribution Licence

\section{References}

1. Carta, R., Sfakiotakis, M., Pateromichelakis, N., Thoné, J., Tsakiris, DP., Puers, R., "A multi-coil inductive powering system for an endoscopic capsule with vibratory actuation". Sensors and Actuators A: Physical, 172, 2011, pp. 253-258.

2. Vilaa, JL., Sallan, J., Llombart, A., Sanz, JF., "Design of a high frequency inductively coupled power transfer system for electric vehicle battery charge". Applied Energy, 86(3), 2009, pp.355-363.

3. Iero, D., Isernia, T., Morabito, AF., Catapano, I., Crocco, L., "Optimal constrained field focusing for focusing for hyperthermia cancer therapy: a feasibility assessment on realistic phantoms". Progress in Electromagnetics Research, 102, 2010, pp.125-141.

4. Din, NM., Chakrabarty, CK., Bin, I. A., Devi, K., Chen W. Y., "Design of RF energy harvesting system for energizing low power devices". Progress in Electromagnetics Research, 132, 2012, pp.49-69.

5. Shao, Q., Liu, H., Fang, X. L., "Efficiency analysis and optimization of wireless power transfer system for freely moving biomedical implants". Science China Technological Sciences, 60(1), 2017, pp.91-101.

6. Fang, X. L., Liu, H., Li, G. Y., Shao, Q., LI, H. Y., "Circuit model based design and analysis for a four-structure-switchable wireless power transfer system". Science China Technological Sciences, 58(3), 2015, pp.534-544.

7. Fu, W. Z., Zhang, B., Qiu, D. Y., "Frequency tracking resonant coupling power wireless transmission system research". In: Chinese University Electric Power Electronic and Electric Drive Academic Annual Meeting, Beijing, China: IEEE, 2009, pp.55-60.

8. Zhang, X., Yang, Q. X., "Research on the maximum transmission efficiency of resonant-coupled wireless transmission high-speed train system". In: China Electromagnetic Compatibility Conference, Beijing, China: IEEE, 2015, pp.114-120.

9. He, H. B., "Detonation mechanism and control strategy of wireless power transmission system". Master thesis of Xiangtan University, China, 2016, pp.4-69.

10. Fu, W. Z., Zhang, B., Qiu, D. Y., "Study on frequency tracking resonant coupled radio power transmission system". Journal of Inverter World, 8, 2009, pp.41-46.

11. Qiang, H., Huang, X. L., Tan, L. L., "The maximum power transmission of inductive coupled wireless power transmission system based on dynamic tuning". Science China Technological Sciences, 42(7), 2012, pp.830-837.

12. Sun, Y., Wang, Z. H., Dai, X., "Study on frequency stability of non-contact power transmission system". Journal of Electrical Engineering, 20(11), 2005, pp.56-59.

13. Zhu, C. B., Yu, C. L., Mao, Y. H., "Loss analysis of magnetic resonance wireless energy transmission system". Journal of Electrical Engineering, 27(4), 2012, pp.13-17.

14. Wang, C. S., Covic, G. A., Stielau, O. H., "Power transfer capability and bifurcation phenomena of loosely coupled inductive power transfer systems". IEEE Transactions on Industrial Electronics, 51, 2004, pp.148-157.

15. Qiang, H., Huang, X. L., Tan, L. L., "Study on topology design of wireless power transfer for electric vehicle based on magnetic resonance coupling". Advanced Materials Research, 308-310, 2011, pp.1000-1003.

16. Sample, A. P., Meyer, D. A., Smith, J. R., "Experimental results, and range adaptation of magnetically coupled resonators for wireless power transfer". IEEE Transactions on Industrial Electronics, 58(2), 2011, pp.544-554.
17. Budhia, M., Boys, J. T., Covic, G. A., "Development of a singlesided flux magnetic coupler for electric vehicle IPT charging systems". IEEE Transactions on Industrial Electronics, 60(1), 2013, pp.318-328.

18. Zhu, C., Liu, K., Yu, C., "Simulation and experimental analysis on wireless energy transfer based on magnetic resonances". In: Vehicle Power and Propulsion Conference, Harbin, China: IEEE, 2008, pp.1-4.

19. Zhen, N. L., Chinga, R. A., Tseng, R., "Design and test of a highpower high-efficiency loosely coupled planar wireless power transfer system". IEEE Transactions on Industrial Electronics, 56(5), 2009, pp.1801-1812.

20. Casanova. J. J., Zhen, N. L., Lin, J., “A loosely coupled planar wireless power system for multiple receivers". IEEE Transactions on Industrial Electronics, 56(8), 2009, pp.3060-3068.

21. Kim, Y. H., Kang, S. Y., Cheon, S., "Optimization of wireless power transmission through resonant coupling". In: International Symposium on Power Electronics Electrical Drives Automation and Motion Conference, Ohrid, Macedonia: IEEE, 23, 2010, pp.426-431.

22. Villa, J. L., Sallan, J., Osorio, J. F. S., "High-misalignment tolerant compensation topology for ICPT systems". IEEE Transactions on Industrial Electronics, 59(2), 2012, pp.945-951.

23. Kim J., Choi, W. S., Jeong, J., "Loop switching technique for wireless power transfer using magnetic resonance coupling". Progress in Electromagnetics Research, 138, 2013, pp. 197-209.

24. Ricketts, D. S., Chabalko, M. J., Hillenius, A., "Optimization of wireless power transfer for mobile receivers using automatic digital capacitance tuning". In: European Microwave Conference, Nuremberg, Germany: IEEE, 2013, pp. 515-518.

25. Chen, C. J., Chu, T. H., Lin C. L., Jou, Z. C., “A study of loosely coupled coils for wireless power transfer". IEEE Transactions on circuits and systems II, 57, 2010, pp. 536-540.

26. Qiang, H., Huang, X. L., Ji, Q. J., Zhao, J. M., “Achieving maximum power transfer of inductively coupled wireless power transfer system based on dynamic tuning control". Science China Technological Sciences, 55(7), 2012, pp.1886-1893.

27. Yang, F. X., "ICPT-based wireless power transmission network key technology research". PhD thesis of Chongqing University, China, 2012, pp.3-126.

28. Raval, P., Kacprzak, D., Hu, A. P., "A wireless power transfer system for low power electronics charging application". In: Industrial Electronics and Applications Conference, Beijng, China: IEEE, 2011, pp.520-525.

29. Hirai, J. J., Kim, T. W., Kawamura, A., "Wireless transmission of power and information for cableless linear motor drive". IEEE transactions on Power Electronics, 15(1), 2000, pp.21-27.

30. Xia, C. Y., Jia, N., Zhuang, Y. H., "Study on frequency stabilization characteristics of dynamic load ICPT". Journal of Electric Machines and Control, 17 (8), 2013, pp.50-55.

31. Madawala, U. K., Thrimawithana, D. J., Nihal, K., "An ICPTsupercapacitor hybrid system for surge-free power transfer". IEEE Transactions on Industrial Electronics, 54(6), 2007, pp.3287-3297.

32. John, T., Grant, A. J. E., "An appropriate magnetic coupling coefficient for the design and comparison of ICPT pickups". IEEE Transactions on Power Electronics, 22(1), 2007, pp.333-335.

33. Cao, L. L., Chen, Q. H., Ren, X. Y., "Research progress of high efficiency wireless charging technology for electric vehicle". Journal of Electrical Engineering, 27 (8), 2012, pp.1-10. 
34. Zhao, B., Chen, X. Y., Yu, Q. G., “Adaptive resonant technology for non-contact power transmission". New Technology of Electric Power, 29 (2), 2010, pp.33-37.

35. Li. Y., Zhang, Y. X., Yang, Q. X., “Analysis and experimental verification of maximum power efficiency of magnetic coupled resonant radio energy transmission system". Journal of Electrical Engineering, 31 (2), 2016, pp.18-24.
36. Li, Y., Yang, Q. X., Yan, Q., "Analysis of the effective transmission distance of radio energy and its influencing factors". Journal of Electrical Engineering, 28 (1), 2013, pp.106-112. 